\title{
The relationship between androgen levels and NLR in hirsutism
}

\author{
Samet SAYILAN ${ }^{1}$ Meral MERT $^{2}$ Aylin AYDIN SAYILAN ${ }^{3}$ Yildiz OKUTURLAR $^{1}$ Ozlem HARMANKAYA $^{1}$ \\ 1. Bakirkoy Dr. Sadi Konuk Education and Research Hospital Internal Medicine Clinic \\ 2. Bakirkoy Dr. Sadi Konuk Education and Research Hospital Endocrinology Clinic \\ 3. Kirklareli University School of Nursing Department
}

\section{Objectives:}

Hirsutism is a disease in which, women chin, upper lip, chest, abdomen, showing the terminal structure to increase hair growth in the male pattern of distribution as androgen sensitive back and thighs are seen (1). Neutrophil / lymphocyte ratio (NLR) has been proposed as a simple inflammatory marker of response. Neutrophil / lymphocyte ratio (NLR) viewed in blood in recent years, providing valuable information in determining the prognosis and diagnosis of various diseases, measurement has emerged as an easy and practical (2). Increased value of NLR cardiovascular venture in patients was found to be an indicator of poor prognosis (3). In our study, we aimed to determine the association between regardless of the etiology in patients with complaints of hirsutism, androgen levels in blood neutrophil / lymphocyte ratio.

\section{Methods:}

248 patients admitted Hirsutism was examined. Hirsutism and menstrual histories menarche, number of pregnancies and live births and infertility history of individuals, were questioned. In addition, hair loss, acne was recorded as signs of hyperandrogenism. Patients FSH, LH, E2, PRL, DHEAS, 17-OHP, androstenedione, total testosterone, free testosterone and follicle tests were measured phase.

NCSS for statistical analysis (Number Cruncher Statistical System) 2007 (Kaysville, Utah, USA) program was used. Data were analyzed using descriptive statistical methods (mean, standard deviation, median, and frequency ratio), Student's $t$ test, as well as the evaluation of variables showing normal distribution; the normal distribution of the data in the two groups Mann-Whitney $U$ test was used for evaluation. The comparison of qualitative data we used Fisher exact test. Results at $95 \%$ confidence interval and the $p<0.05$ level were evaluated which was significant.

The study included between $16-70$ years (mean age $28.67 \pm 9.29$ years) were enrolled 248 patients. Patients with $(n=73)$ and without $(n=175)$ hipeandrojenemi were compared.

Statistically significant differences between age classification based on androgen levels were found $(p<0.05)$. the normal rate of androgen levels have been found to be significantly higher. The average age of patients with normal androgen levels of $29.67 \pm 9.10$, while it is observed that the average age of $26.58 \pm 9.40$ in patients with high.

When the groups with and without hyperandrogenemia are compared there occured no statistically significant difference between the NLR ( $p>0.05$ ). In contrast, significant differences were found between hematocrit levels $(p<0.05)$.

According to androgen levels it was statistically significant differences between ferritin levels ( $p$ $<0.05)$.

Text the presence of hyperandrogenism in hirsutism clinical practice cases are being investigated. The relationship between hyperandrogenism and a parameter NLO which is an early used parameter may be guider to the detection of inflammatory or cardiovascular risk 